\title{
Three-dimensional technologies used for patient specific applications in orthopedics
}

\author{
Flaviu Moldovan ${ }^{*}$, Tiberiu Bataga ${ }^{2}$ \\ 1. IOSUD Doctoral School, George Emil Palade University of Medicine, Pharmacy, Science, and Technology of Targu Mures, Romania \\ 2. Department of Orthopedics - Traumatology, George Emil Palade University of Medicine, Pharmacy, Science, and Technology of Targu Mures, Romania
}

Background: Three-dimensional (3D) technologies have numerous medical applications and have gained a lot of interest in medical world. After the advent of three-dimensional printing technology, and especially in last decade, orthopedic surgeons began to apply this innovative technology in almost all areas of orthopedic traumatic surgery. Objective: The aim of this paper is to give an overview of 3D technologies current usage in orthopedic surgery for patient specific applications. Methods: Two major databases PubMed and Web of Science were explored for content description and applications of 3D technologies in orthopedic surgery. It was considered papers presenting controlled studies and series of cases that include descriptions of 3D technologies compatible with applications to human medical purposes. Results: First it is presented the available three-dimensional technologies that can be used in orthopedic surgery as well as methods of integration in order to achieve the desired medical application for patient specific orthopedics. Technology starts with medical images acquisition, followed by design, numerical simulation, and printing. Then it is described the state of the art clinical applications of 3D technologies in orthopedics, by selecting the latest reported articles in medical literature. It is focused on preoperative visualization and planning, trauma, injuries, elective orthopedic surgery, guides and customized surgical instrumentation, implants, orthopedic fixators, orthoses and prostheses. Conclusion: The new 3D digital technologies are revolutionizing orthopedic clinical practices. The vast potential of 3D technologies is increasingly used in clinical practice. These technologies provide useful tools for clinical environment: accurate preoperative planning for cases of complex trauma and elective cases, personalized surgical instruments and personalized implants. There is a need to further explore the vast potential of 3D technologies in many other areas of orthopedics and to accommodate healthcare professionals with these technologies, as well as to study their effectiveness compared to conventional methods.

Keywords: orthopedic surgery, three-dimensional technologies, 3D printing, clinical workflow, patient-specific applications

Received 5 May 2021 / Accepted 20 June 2021

\section{Introduction}

Continuous improvement of three-dimensional (3D) technologies performance contributes to increasing the number of applications in personalized orthopedic therapy. The new three-dimensional digital technologies have a high potential for use in clinical practice and to increase the efficiency of various medical investigations [1]. Data acquired from medical images are processed with support of specialized software products, which facilitate identification of the analyzed tissues and extraction of specific information. Thus, by processing medical images it can be computerassisted designed patient-specific devices which fit its anatomy. These are then tested with finite element analysis and printed in 3D. In fact these technologies constitute a 3D toolkit that can be integrated into orthopedics and allow effective diagnosis as well as various patient-specific applications in orthopedics [2].

The aim of this paper is to give an overview of $3 \mathrm{D}$ technologies current usage in orthopedic surgery for patient specific applications. It is based on existing literature review. Findings are structured in two parts describing the available three-dimensional technologies for medical applications and then the main clinical applications in orthopedics. It demonstrates how digital information is trans-

* Correspondence to: Flaviu Moldovan

E-mail: moldovan.flaviu95@yahoo.com formed into physical models that imitate actual organs which allow various applications in orthopedic surgery.

\section{Methods}

The research methodology surveys three-dimensional technologies applications by presenting recent developments and usage in medicine as well as limitations of 3D technologies reported in scientific literature. We started from the objectives and goals of 3D technologies medical applications in orthopedic surgery. The systematic review on scientific literature was conducted in PubMed and Web of Science databases. The search strategy has been extensively extended to recent published papers in order to ensure that relevant current work in the field has not been excluded. We searched for relevant keywords in fields of 3D technology and personalized orthopedic surgery. We had also analyzed relevant articles found in references of the selected articles. The databases exploration was carried out between February and April 2021. We considered only complete works presenting controlled studies and series of cases, written in English and German, in which 3D technologies for orthopedic surgical purposes are applied to living people. Manual processing of titles and contents was performed only for papers that include descriptions of 3D technologies compatible with application to human medical purposes.

Findings present in the first part the state of the art available three-dimensional technologies that can be used 
in orthopedic surgery as well as methods of integration in order to achieve the desired medical application in patient specific orthopedics. Second part describes the state of the art clinical applications of 3D technologies in orthopedics by selecting the latest reported articles in medical literature. It is focused on preoperative visualization and planning, injuries, elective orthopedic surgery, guides and customized surgical instrumentation, implants, orthopedic fixatives, orthoses and prostheses.

\section{Available three-dimensional technologies}

The main available 3D technologies refer to 3D imaging and image acquisition, 3D design, 3D numerical simulation, 3D printing, which require a method of integration into a clinical workflow.

\section{D imaging and image acquisition}

Three-dimenional imaging is used for acquisition of technical images such as computed tomography or magnetic resonance imaging and ultrasound. Files are in DICOM (Digital Imaging and Communications in Medicine) format characterized by resolution, which is described in pixels and /or voxels and expressed in Hounsfield units (HU).
Through a segmentation process it provides raw data for extraction of useful information about tissues (figure 1).

\section{D design}

The studied anatomical tissues can be differentiated using a segmentation process and can be transferred to Computer Aided Design (CAD) environment as different geometric entities. These are described by points, which form triangles that represent specific surfaces (figure 2). CAD contains design functions that can be used in virtual surgical simulations, such as: measurements, explorations of anatomical shapes, geometric optimizations, planning of cutting paths, but also design of implants, tools and customized medical devices.

\section{D numerical simulation}

Further, from CAD environment, the anatomical tissues can be transferred as different geometric entities in a finite element analysis environment [3]. These are considered physical systems to which characteristic material properties can be attributed. In this way computer simulations of bone structures can be performed. Also it is allowed application of forces and study of internal tensions, for example

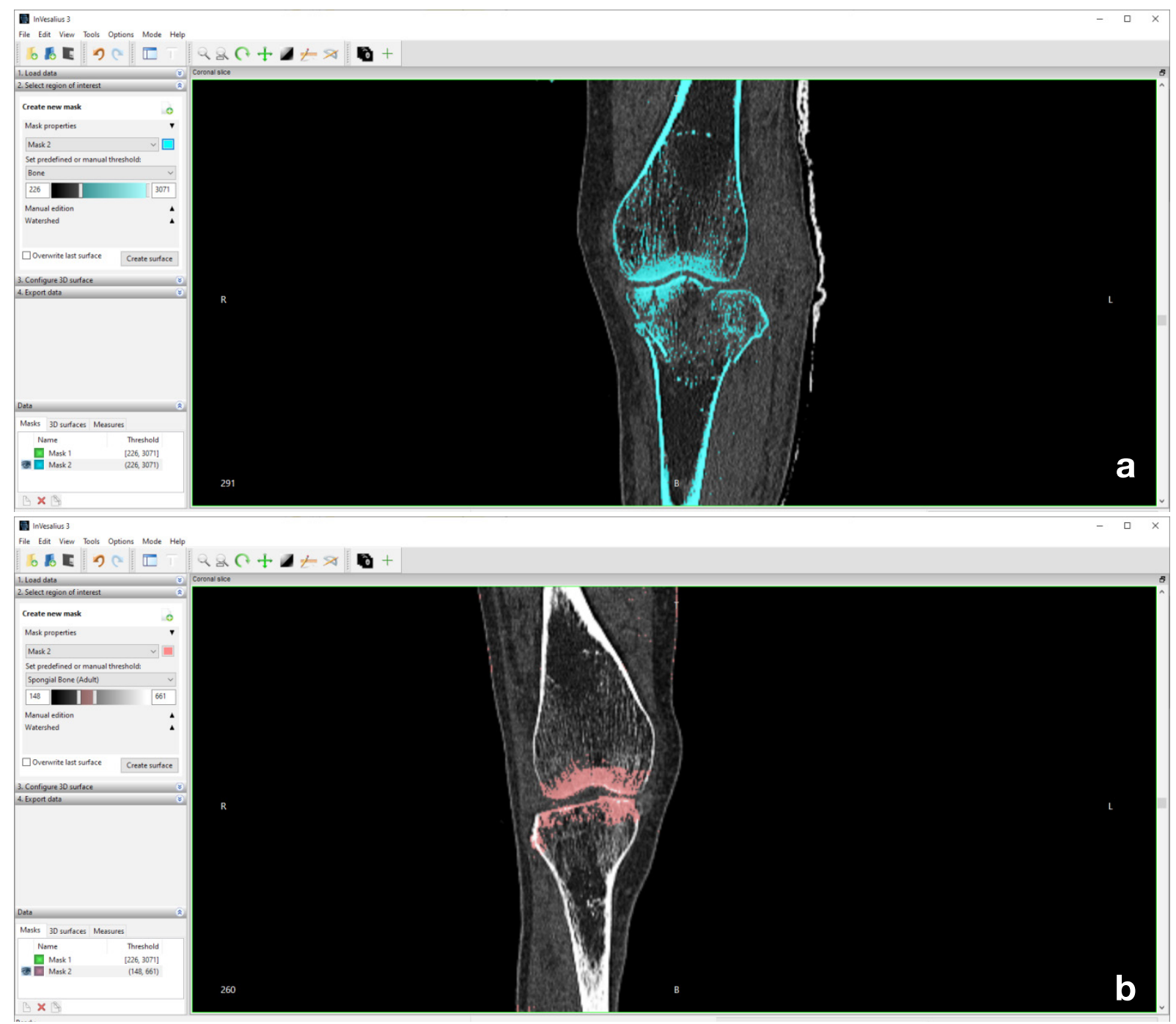

Fig. 1. The contour segmentation process in Invesaliu software with different resolutions: (a) Femur and tibia bones coronal view; (b) Spongial bone threshold. 

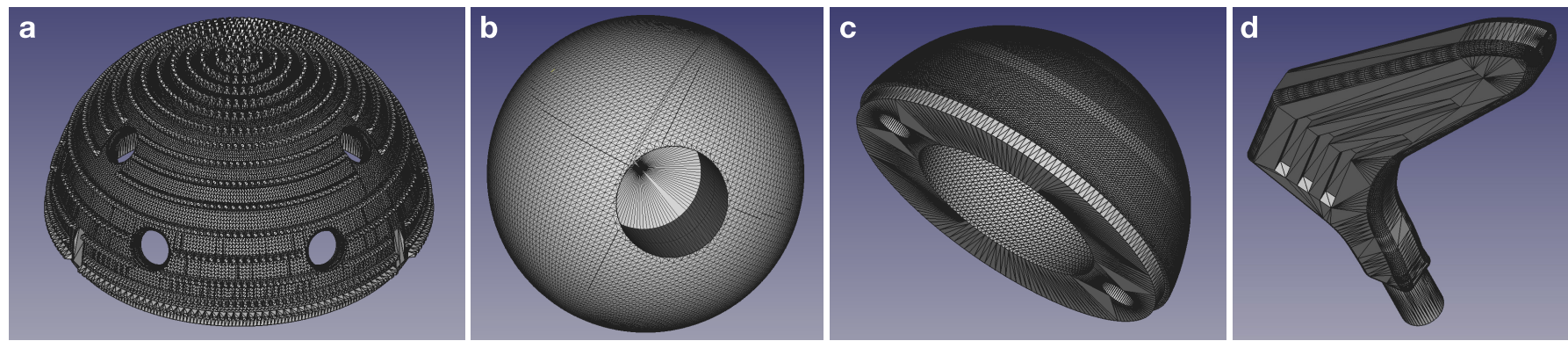

Fig. 2. Custom component of the hip implant 3D computer model: (a) Acetabular shell; (b) Femoral head; (c) Acetabular liner; (d) Femural stem.

studies of effort distribution on the bone surface with loading and reaction force in center location (figure 3).

\section{D printing}

Three-dimensional printing is known also as additive manufacturing or rapid prototyping. Supported by 3D printing it can be manufactured replicas of the patient's anatomy, custom surgical instruments, or custom implants.

Three-dimensional printing is a fast and accurate way to manufacture 3D objects by placing successive thin layers of raw material. In a few hours, objects of high complexity can be built, which combine several materials, colors and textures. These are obtained in desired shape without need for further processing. This technology significantly reduces manufacturing cycle time of the product. Objects are printed based on information from digital 3D files, after being designed with CAD and simulated with finite element analysis.

Most 3D printing technologies work on the basis of one of the following four manufacturing methods: light curing which selectively solidifies a liquid resin; extrusion forming layers of the object by exiting a semi-liquid material from a print-head nozzle; techniques for aggregating powder particles; lamination processes that stick together thin layers of construction material (figure 4).

Each of the presented technologies can be used for different clinical applications, for production of anatomical replicas, customized surgical instruments, surgical guides, as well as implants.

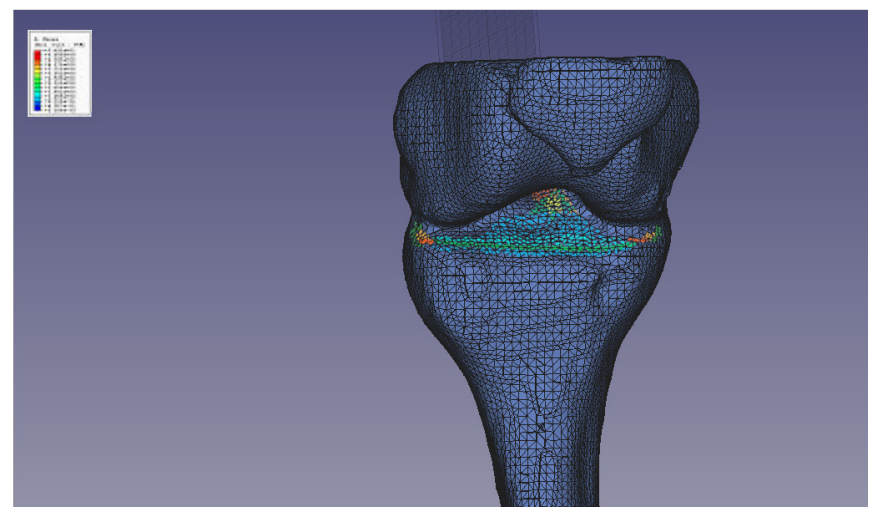

Fig. 3. Distribution of efforts on the bone surface with loading and reaction force in center location.
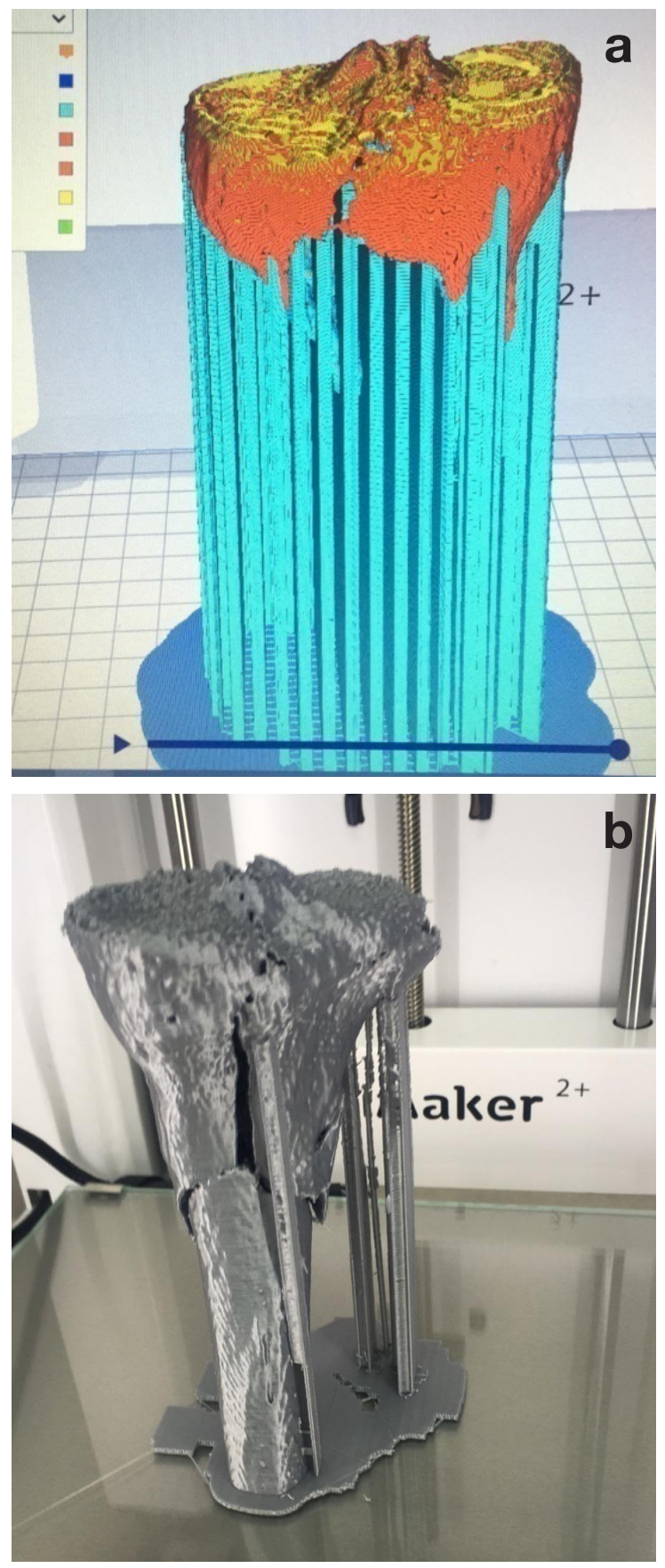

Fig. 4. 3D printing of the tibial plateau fracture model through a Fused Deposition Modeling manufacturing process: (a) Preparation for printing in Ultimaker Cura software; (b) Printed bone replica supported by pillars and a platform using an Ultimaker $2+$ printing machine. 


\section{Integration of technologies in clinical workflows}

In order to be used in clinical applications technologies presented above need to be integrated in several stages leading to the desired purpose for manufacturing customized surgical instruments. By exploring applications of 3D technologies in orthopedic surgery, Moldovan et al [4] have created a clinical workflow for patient-specific applications in orthopedics which integrates $3 \mathrm{D}$ technologies in a sequence described in figure 5 .

The first stage consists in images acquisition from computed tomography and/or magnetic resonance imaging. These files are in DICOM format and are further processed with specialized software. Then it is allowed the extraction of specific information characterizing the studied tissues. This step is performed by orthopedic surgeons. Further, CAD modeling allows design of customized medical objects performed by medical engineers in the form of medical devices and implants. These are numerically simulated for resistance and are validated in a finite element analysis,

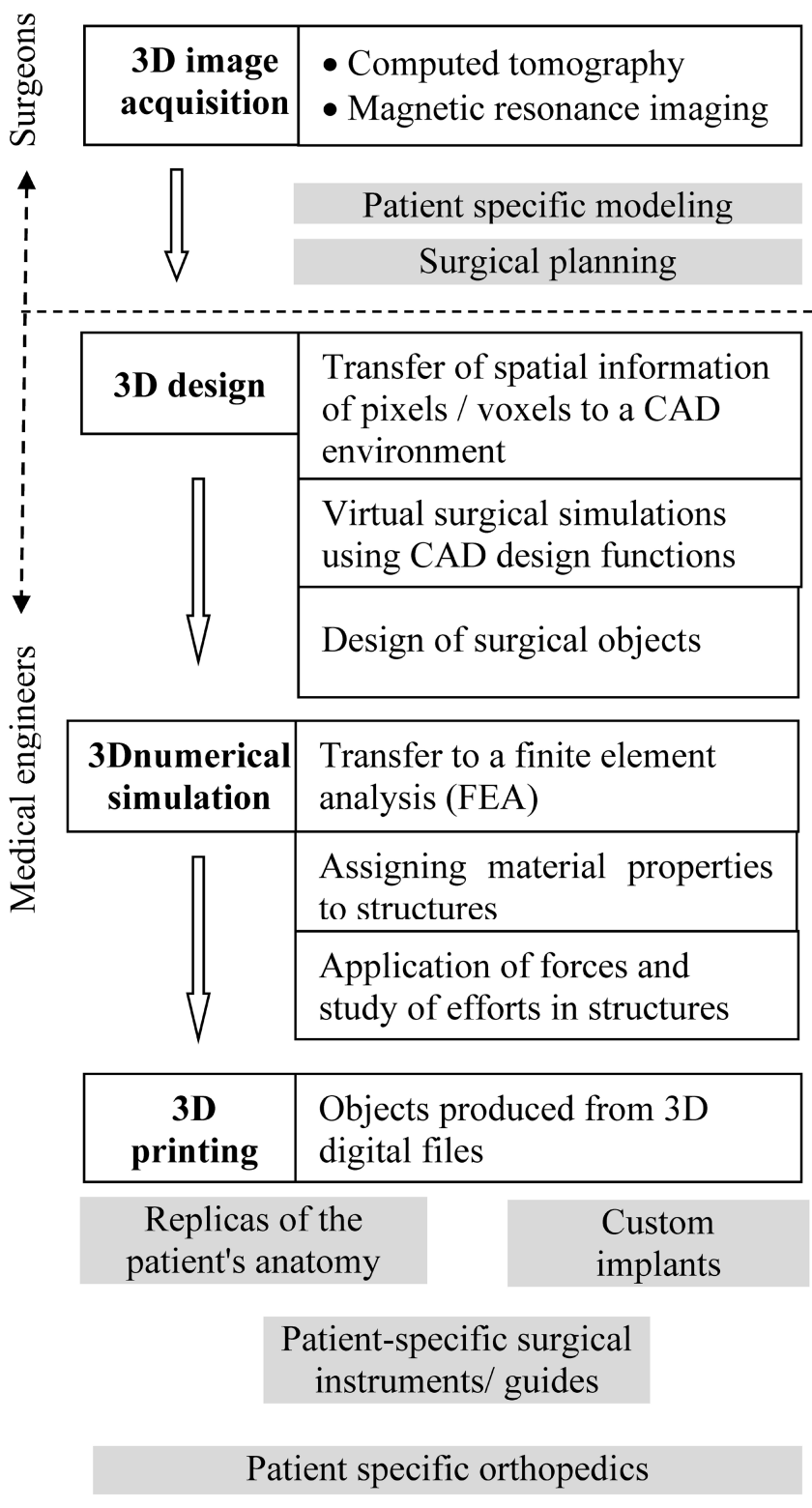

Fig. 5. Clinical workflow integrating 3D technologies for patientspecific applications in orthopedics. by assignment of material properties to structures, application of forces and study of induced efforts. In the end, $3 \mathrm{D}$ printing physically builds custom medical objects that were previously designed, which may consist in replicas of the patient's anatomy, custom implants, patient specific surgical instruments and/or guides.

\section{Clinical applications in orthopedics}

There are many digital solutions that help orthopedic surgeons. This is already a quite vast field, that is expanding rapidly and it is difficult to get a complete overview of existing solutions [5]. Three-dimensional technologies are revolutionizing preoperative planning for many orthopedic procedures [6], from complicated trauma cases [7] to elective orthopedic surgery [8]. They assist surgeons with $3 \mathrm{D}$ visualizations of the anatomy and allow construction of accurate 3D anatomical replicas.

\section{Preoperative visualization and planning}

Three-dimensional printing technology has been used successfully in preoperative planning of complex acetabular fractures [9], clavicle fractures [10], tibial fractures [11], pelvic surgery [12], trimalleolar and calcaneal fractures [13], elbow fractures [14], complicated proximal shoulder fractures [15], ankle fractures [16], rib fractures [17], long bone fractures [18], as well as in pre-contouring of fixation plates [19].

Three-dimensional printing technology used in musculoskeletal surgery has a huge technical, medical and economic potential, which is of interest to all those involved in the treatment of musculoskeletal problems [20]. In a meta-study Morgan et al [21] showed that use of 3D printing in preoperative planning of orthopedic trauma reduces operating time with $19.85 \%$, reduces intraoperative blood loss with $25.73 \%$ and the number of fluoroscopy uses decreases with $23.80 \%$.

\section{Injuries}

Three-dimensional printing of joint fractures is an innovative procedure that generates useful models in preoperative, tangible and real assessment of fractures and surgical procedures, which improves provided care and has the potential to raise the patient's understanding and agreement [22].

Bagaria et al [7] presented a series of cases in which they used computed tomography / magnetic resonance imaging medical scans, 3D reconstruction, anatomical modeling, computer-aided design and computer-assisted implantation in treatment of complex fractures of acetabulum, calcaneus and medial condyle of the femur (Hoffa's fracture). Chung et al [23] presented in scientific literature the use of a 3D printed calcaneus model as a preoperative and intraoperative tool for minimally invasive fixation of a plate on the calcaneus fracture. Subsequently, Yao et al [24], using a 3D printed heel model, performed an intervention in which they simulated the fixation with screws in the minimally invasive treatment 
of heel fractures. Bizzotto et al [25] have used 3D printed medical models to assess fragment dislocation and joint surface failure, which were collected over a two-year period from 102 patients diagnosed with complex joint fractures of distal radius, radial head, tibial plateau, astragalus, calcaneus, ankle, humeral head and glenoid. In addition, models were presented to patients in order to obtain informed consent before surgery.

With the support of 3D technologies, an innovative research carried out by Moldovan et al [26], has led to elaboration of a new alignment method for bone fragments in comminutive fractures. The new algorithm is able to align bone fragments surfaces derived from segmentation process of volumetric tomographic data. The method was validated in practice for preoperative planning of a 49-year-old male patient who had a tibial plateau fracture of Schatzker type VI (figure 6).

\section{Elective orthopedic surgery}

Computer-assisted three-dimensional preoperative planning for elective orthopedic cases was performed in spinal disorders [27, 28], sports medicine [29], adult reconstruction $[30,31]$, foot and ankle surgery $[32,33]$, pediatric orthopedics [34, 35], and orthopedic oncology [36, 37].

Li et al. [27] compared intervention in two groups of patients undergoing lumbar discectomy for whom classical preoperative planning was performed, with preoperative planning supported by $3 \mathrm{D}$ printing technology through which patient-specific lumbar vertebral models were created. A comparison of preoperative and postoperative clinical outcomes of the two groups found that use of $3 \mathrm{D}$ printing technology reduced duration of surgery and perioperative blood loss, but clinical outcomes were similar for the two groups of patients. In a case of 50 patients diagnosed with upper thoracic fracture, Kaya et al [28] inserted a pedicular screw using $3 \mathrm{D}$ printed guide plates, which were produced cheaply and customized for each patient. The new method allowed a safe surgery combined with high precision insertion of thoracic pedicle screws.

With the support of 3D printing Sheth et al [29] produced a 3D model of the glenohumeral joint of a young patient with recurrent anterior shoulder instability and complex Bankart and Hill-Sachs lesions. It was used in preoperative planning stages of a Bankart arthroscopic repair and completion to determine depth of the Hill-Sachs lesion as well as degree of abduction and external rotation caused by the Hill-Sachs lesion.

A retrospective study by Liang et al [30] reports the feasibility of using 3D printing technology for 35 patients with pelvic tumor. They underwent resection of pelvic tumor and reconstruction using 3D printed endoprostheses. Three-dimensional printing technology has facilitated precise matching and osseointegration between implant and host bone. Hun et al [31] describe the successful use of custom $3 \mathrm{D}$ printed prostheses in complex spine surgery for $\mathrm{C} 2$ reconstruction of the anterior spine. Three-dimensional
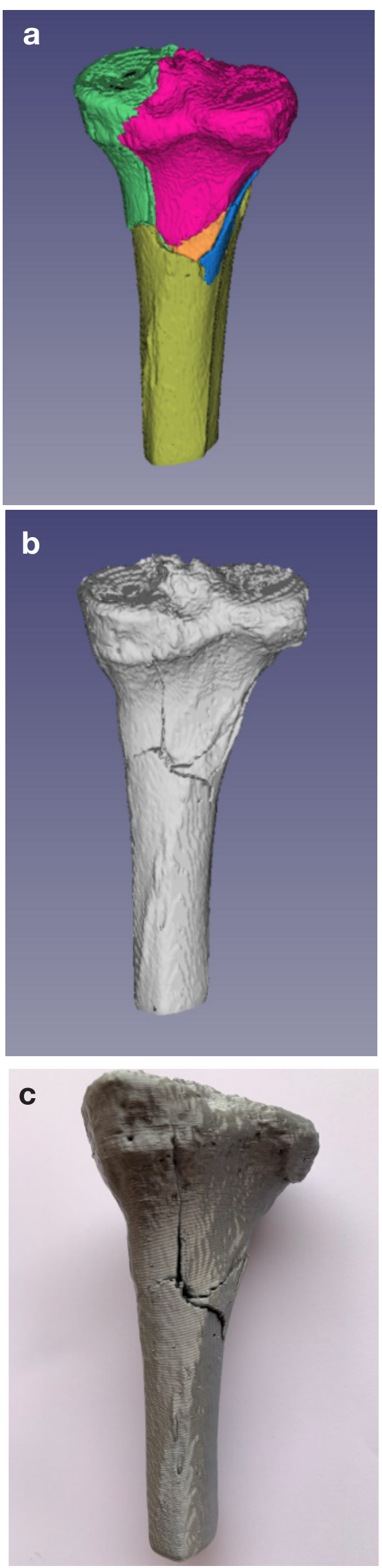

Fig. 6. Tibial plateau fracture reconstruction result supported by the alignment algorithm: (a) Identification of independent fracture fragments; (b) Standard triangle language model; (c) Three-dimensional printed replica. 
printed, patient-specific titanium prostheses were used for reconstruction of structurally compromised $\mathrm{C} 2$ vertebrae.

Reconstruction of the foot and ankle is often complicated by multiplanar deformity and bone loss. Threedimensional printing technology offers solutions to these complex cases with customized implants conforming to the anatomy and patient-specific tools that allow precise correction of the deformation. Kadakia et al [32] treated four cases of complex reconstruction of the lower extremities characterized by segmental bone loss and deformation with failed total arthroplasty of the ankle, avascular necrosis of the talus, ballistic trauma and failure of a tibial osteotomy. Each case presented a unique clinical scenario for which 3D printing technology allowed innovative solutions. By employment of 3D printing technology, Zhang et al [33] treated symptomatic bone cysts of the ankle and foot, using $3 \mathrm{D}$ printed models to position the cysts, which facilitated an arthroscopic approach, a better understanding of the anatomy and easy operation.

Starosolski et al [34] have used 3D biomedical models for evaluation of pediatric musculoskeletal disorders in a case of Perthes disease, which allowed deformity precise assessment, exact placement of implants and procedure simulation. For three young patients, Coote et al [35] have used 3D printed models of patients' spine based on computed tomography in pediatric spine deformity operations that allowed an efficient and accurate surgical planning by taking into account anatomical complexity and risk of devastating neurological consequences.

Ren et al [36] printed a heel 3D model of a 17-yearold patient, with a history of nocturnal ankle pain, which allowed precise location of an osteoid osteoma. In the complex context of foot anatomy, this challenge facilitated the design of a surgical guide plate, which guided intraoperatively a trephine, and created a bony window in the lateral area of the calcaneus, through which, with minimal bone loss, it was achieved a complete lesion resection. Three-dimensional printing technology provides an advanced approach to pelvic bone tumor resection and reconstruction. Park et al [37] performed malignant pelvic bone tumor surgeries for 12 patients using bone cutting guides and $3 \mathrm{D}$ printed implants. It was performed a type I internal hemipelvectomy by using $3 \mathrm{D}$ printed bone cutting guides that underwent bone tumor resection in the ileum cavitary, and type I, II, and III pelvic resections by using $3 \mathrm{D}$ printed cutting guides and reconstruction with $3 \mathrm{D}$ printed implants.

\section{Guides and customized surgical instrumentation}

After preoperative planning, as previously presented, 3D technology can still be used in manufacture of customized surgical guides and instruments, such as templates and cutting tools. The use of personalized surgical instruments optimizes patient's functional results, reduces duration of surgery and perioperative blood loss [38].
Most often, patient-specific instrumentation is used to completely replace the knee. With the support of $3 \mathrm{D}$ technology, knee models are printed and then disposable cutting tools adapted to the anatomy of the patient's knee are manufactured, which minimizes potential surgical errors and optimizes restoration of the knee mechanical axis [39]. However, Nam et al [40], after two years of clinical experience in total knee arthroplasty, show that customized cutting guides that use the preoperative $3 \mathrm{D}$ imaging for fabrication of cutting blocks specific to the patient's native anatomy, compared with standard instruments do not demonstrate advantages in knee result measurement scores or coronal alignment.

Recently, the use of custom 3D printed instruments has been extended beyond total knee replacement, to other custom surgeries. Hirao et al [41] treated a patient with rheumatoid arthritis who had a degeneration of the first metatarsophalangeal joint and fused in a severe position of pronation deformity. In an arthrodesis operation of the metatarsophalangeal joint, a personalized surgical guide was used, constructed after 3D simulation, which allowed the joint to be placed in an appropriate rotational position by adjusting the rotation between the metatarsal and the proximal phalanx. Nineteen children with forearm deformity who were diagnosed by Bauer et al [42] with hereditary multiple exostoses, Madelung's deformity, and healed fractures with incorrect anatomical alignment, underwent corrective osteotomies. It was used preoperative $3 \mathrm{D}$ computer modeling and specific patient surgical guidelines.

In a case of 12 patients, Arnal-Burró et al [43] have used a new technique based on printed 3D cutting guides and wedge spacers for distal femoral osteotomies. Results were compared with 20 control patients who underwent distal femoral osteotomies with opening wedges following the traditional technique. In comparison with control group the use of customized 3D guides has minimized intraoperative complications, while reducing surgical and fluoroscopic time, as well as costs. IJpma et al [44] used patient-specific osteosynthesis plates with drilling guides for operative treatment of patients with displaced acetabular fractures. The surgery main results were the quality of the reduction. The secondary results consisted in accuracy of screws placement and clinical result reported by the patient.

In a case of a giant invasive sacral schwannom, for osteotomy Lin et al [45] have used a digital model of the sacrum for 3D printing of a customized guiding tool which helped them to perform the appropriate tumor resection with precise excision edges. For precise resection of osteosarcoma, Ma et al [46] have used patient-specific guidance templates that led during surgery to more accurate resection of tumor bone and bone implants, reduced blood loss, shorter duration of surgery, and reduced radiation exposure. 


\section{Implants}

Customized 3D printed implants are mainly used in reconstructive surgery $[47,48]$ and orthopedic oncology $[49,50]$ because their shape matches excised bone, thus lowering stresses on the existing bone compared to conventional implants.

In total hip arthroplasties with severe acetabular bone defects, 3D printed implants customized to each patient's bone defect provide stable fixation and improved hip scores. Li et al [47], who treated Paprosky type IIIB defects, reported improvements in the mean Harris score of the hip between preoperative and postoperative, from 36 to 82 points. This system can be considered an effective treatment option in almost all cases of acetabular bone loss, except for those of pelvic discontinuity. After total shoulder arthroplasty glenoid component damage is one of the most common major complications. Stoffelen et al [48] performed surgery in which, after a thorough debridement of soft tissues, they introduced bone autografts supported and fixed successfully a titanium glenoid implant in affected shoulder glenoid component.

In a case of a patient with grade 2 calcaneus chondrosarcoma, Imanishi and Choong [49] performed a total calcanectomy by replacing the calcaneus with a customized patient calcanian prosthesis which was $3 \mathrm{D}$ printed in titanium. In a research of Chandhanayingyong et al [50], a patient who had a destructive lesion of the toe 5 proximal phalanx with invasion of the metatarsophalangeal joints and was diagnosed with giant bone cell tumor grade 1 underwent a block resection of the proximal phalanx. The defect was reconstructed with a proximal phalanx endoprosthesis as a metatarsophalangeal joint extension, which was $3 \mathrm{D}$ printed in titanium.

\section{Orthopedic fixatives, orthoses and prostheses}

Qiao et al [51] combined 3D printing with computerassisted reduction technique in order to develop a custom external fixator with fracture reduction function, similar to Ilizarov model, which was used to treat three patients diagnosed with tibial diaphyseal fractures. For three fracture models, reduction and fixation were performed by connecting pins inserted in bones supported by the custom fixator. The custom external fixator has advantages in easy handling and no experience required. Good reduction results were obtained in all three cases in terms of exact reduction, proper fixation, minimal invasion, joint fracture fragmentation and pain.

In a case of 29 children diagnosed with moderate adolescent idiopathic scoliosis, Raux et al [52] have used orthopedic treatment performed with traditional torso surface acquisition method based on a plaster matrix compared to the computer-aided design method with 3D reconstruction of the trunk by optical scanning and $3 \mathrm{D}$ printing for the manufacture of corrective devices. Each patient received a corrective device manufactured by the two technologies and selected a device according to their own comfort. It was preferred 13 devices manufactured by classical technology and 16 three-dimensional printed devices. Both technologies provided good results in terms of correction made and in terms of patient comfort with correction devices. Three-dimensional printing had the advantage of a less invasive and more hygienic procedure. Subsequently, in a clinical evaluation, Cobetto et al [53] demonstrated better efficacy of correction devices that are designed and manufactured computer-assisted and finiteelement simulated, compared to those that do not benefit from finite-element simulation, on two groups of 19 and 21 patients diagnosed with scoliosis. The simulated rib cages allowed better results of the main thoracic and lumbar curves. These were 50\% thinner and had 20\% less coverage, compared to cages unsimulated with finite elements.

Dombroski et al [54] conducted a study to compare custom orthoses made of traditionally cast plaster foot molds, respectively $3 \mathrm{D}$ printed. Results evaluation consisted in medial longitudinal arch measurement using arch height index during 10 walking cycles, which highlighted slightly improved results in the case of 3D printed orthoses.

According to a review of Tanaka and Lightdale-Miric [55], advances in 3D technology allows manufacture of custom prosthetic hands at much more affordable costs, which are about 70 times lower than traditionally manufactured. Using 3D technology, Swartz et al [56] fabricated prosthesis in a case of a partially amputated hand on index finger and non-dominant left thumb. The prosthesis also contained a control device that compared to traditional hand prostheses offered greater strength and functionality and a smaller overall size.

In a case of an 8-year-old child with bilateral hand surgical amputation, Galvez et al [57] selected the most appropriate size for hand transplantation using $3 \mathrm{D}$ printed models from different potential donors for which they determined depending on size hands and forearms matching between donor and recipient.

\section{Conclusion}

From the scientific literature analysis it can be seen that new 3D digital technologies are revolutionizing orthopedic clinical practices. In recent years, the vast potential of these technologies, which include 3D imaging, design, numerical simulation in design and 3D printing, is increasingly used in clinical practice. The first and most important step is to acquire accurate image data. The obtained data are then imported and processed by specialized image processing software packages in order to identify and extract specific information about tissues.

These technologies have the potential to provide useful tools for clinical environment, because complex structures such as patient anatomy replicas, custom surgical instruments, custom implants, but also other medical devices can be manufactured in a few hours. Visualization of 3D printed anatomical models and preoperative education of patients can help orthopedic surgeons in selecting the op- 
timal operating plan, making implants in the most appropriate positions which optimizes clinical results and minimizes complications.

Scientific literature research has shown that in many surgical situations, by means of $3 \mathrm{D}$ technologies, accurate preoperative planning can be achieved in cases of complex trauma as well as elective cases. Supported by 3D technologies personalized surgical instruments and personalized implants have been developed, especially in reconstruction interventions for adults and in orthopedic oncology. By using personalized patient guidance tools, surgery in complex cases is easier to perform, more accurate and costeffective, even if personalized implants and patient-specific instruments can be costly.

Three-dimensional technologies have a valuable potential that requires exploration for their effective integration and application in clinical practice. The vast potential of 3D technologies can still be applied in many other areas of orthopedics. In future research it should be compared patient outcomes and effectiveness of preoperatively planned interventions supported by conventional methods with those using 3D technology. Physicians should also adapt to and learn these technologies in order to support their implementation in clinical practice and to perform further research.

\section{References}

1. Lal H, Patralekh MK. 3D printing and its applications in orthopaedic trauma: A technological marvel. J Clin Orthop Trauma. 2018;9(3):260268.

2. Papagelopoulos PJ, Savvidou OD, Koutsouradis P, Chloros GD, Bolia IK, Sakellariou VI, Kontogeorgakos VA, Mavrodontis II, Mavrogenis AF, Diamantopoulos P. Three-dimensional Technologies in Orthopedics. Orthopedics. 2018;41(1):12-20.

3. Pfeiffer FM. The Use of Finite Element Analysis to Enhance Research and Clinical Practice in Orthopedics. J Knee Surg. 2016;29(2):149-58.

4. Moldovan F, Gligor A, Bataga T. Integration of Three-dimensional Technologies in Orthopedics: A Tool for Preoperative Planning of Tibial Plateau Fractures. Acta Inform Med. 2020;28(4):278-282.

5. Braun BJ, Grimm B, Hanflik AM, Marmor MT, Richter PH, Sands AK, Sivananthan S. Finding NEEMO: towards organizing smart digital solutions in orthopaedic trauma surgery. EFORT Open Rev. 2020;5(7):408-420.

6. Segaran N, Saini G, Mayer JL, Naidu S, Patel I, Alzubaidi S, Oklu R. Application of 3D Printing in Preoperative Planning. J Clin Med. 2021;10(5):917.

7. Bagaria V, Deshpande S, Rasalkar DD, Kuthe A, Paunipagar BK. Use of rapid prototyping and three-dimensional reconstruction modeling in the management of complex fractures. Eur J Radiol. 2011;80(3):814-20.

8. Zhong H, Ma S, Cen Y, Ma L, Li D, Liang B, Chen J, Zhang Y. A case report of early unilateral external fixation by $3 \mathrm{D}$ printing and computerassisted and secondary bone graft internal fixation in pseudarthrosis of the tibia surgery. J Int Med Res. 2020;48(9):300060520945518.

9. Wang C, Chen Y, Wang L, Wang D, Gu C, Lin X, Liu H, Chen J, Wen X, Liu Y, Huang F, Yao L, Fan S, Huang W, Dong J. Three-dimensional printing of patient-specific plates for the treatment of acetabular fractures involving quadrilateral plate disruption. BMC Musculoskelet Disord. 2020;21(1):451.

10. Kim HN, Liu XN, Noh KC. Use of a real-size 3D-printed model as a preoperative and intraoperative tool for minimally invasive plating of comminuted midshaft clavicle fractures. J Orthop Surg Res. 2015;10:91.

11. Liu P, Hewitt N, Shadid W, Willis A. A system for 3D reconstruction of comminuted tibial plafond bone fractures. Comput Med Imaging Graph. 2021;89:101884.

12. Wang J, Wang X, Wang B, Xie L, Zheng W, Chen H, Cai L. Comparison of the feasibility of $3 D$ printing technology in the treatment of pelvic fractures: a systematic review and meta-analysis of randomized controlled trials and prospective comparative studies. Eur J Trauma Emerg Surg. 2020; Epub ahead of print.

13. Yang L, Shang XW, Fan JN, He ZX, Wang JJ, Liu M, Zhuang Y, Ye C. Application of $3 \mathrm{D}$ Printing in the Surgical Planning of Trimalleolar Fracture and Doctor-Patient Communication. Biomed Res Int. 2016;2016:2482086.

14. Yang L, Grottkau B, He Z, Ye C. Three dimensional printing technology and materials for treatment of elbow fractures. Int Orthop. 2017;41(11):2381-2387.

15. Campana V, Cardona V, Vismara V, Monteleone AS, Piazza P, Messinese P, Mocini F, Sircana G, Maccauro G, Saccomanno MF. 3D printing in shoulder surgery. Orthop Rev (Pavia). 2020;12(Suppl 1):8681.

16. Jastifer JR, Gustafson PA. Three-Dimensional Printing and Surgical Simulation for Preoperative Planning of Deformity Correction in Foot and Ankle Surgery. J Foot Ankle Surg. 2017;56(1):191-195.

17. Zhou X, Zhang D, Xie Z, Yang Y, Chen M, Liang Z, Zhang G, Li S. Application of $3 \mathrm{D}$ printing and framework internal fixation technology for high complex rib fractures. J Cardiothorac Surg. 2021;16(1):5.

18. Liodakis E, Bruns N, Macke C, Krettek C, Omar M. 3D-Druck-Templategestützte Reposition von Frakturen der langen Röhrenknochen [3D-printed template-assisted reduction of long bone fractures]. Unfallchirurg. 2019;122(4):286-292.

19. Marinescu R, Popescu D, Laptoiu D. A Review on 3D-Printed Templates for Precontouring Fixation Plates in Orthopedic Surgery. J Clin Med. 2020;9(9):2908.

20. Krettek C, Bruns N. Aktueller Stand und neue Entwicklungen des 3D-Drucks in der Unfallchirurgie [Current concepts and new developments of $3 \mathrm{D}$ printing in trauma surgery]. Unfallchirurg. 2019;122(4):256-269.

21. Morgan C, Khatri C, Hanna SA, Ashrafian H, Sarraf KM. Use of threedimensional printing in preoperative planning in orthopaedic trauma surgery: A systematic review and meta-analysis. World J Orthop. 2020;11(1):57-67.

22. Chen C, Cai L, Zheng W, Wang J, Guo X, Chen H. The efficacy of using 3D printing models in the treatment of fractures: a randomised clinical trial. BMC Musculoskelet Disord. 2019;20(1):65.

23. Chung KJ, Hong DY, Kim YT, Yang I, Park YW, Kim HN. Preshaping plates for minimally invasive fixation of calcaneal fractures using a realsize 3D-printed model as a preoperative and intraoperative tool. Foot Ankle Int. 2014;35(11):1231-6.

24. Yao LF, Wang HQ, Zhang F, Wang LP, Dong JH. Minimally invasive treatment of calcaneal fractures via the sinus tarsi approach based on a 3D printing technique. Math Biosci Eng. 2019;16(3):1597-1610.

25. Bizzotto N, Tami I, Santucci A, Adani R, Poggi P, Romani D, Carpeggiani G, Ferraro F, Festa S, Magnan B. 3D Printed replica of articular fractures for surgical planning and patient consent: a two years multi-centric experience. 3D Print Med. 2015;2(1):2.

26. Moldovan F, Gligor A, Bataga T. Structured Integration and Alignment Algorithm: A Tool for Personalized Surgical Treatment of Tibial Plateau Fractures. J Pers Med. 2021;11(3):190.

27. Li C, Yang M, Xie Y, Chen Z, Wang C, Bai Y, Zhu X, Li M. Application of the polystyrene model made by $3-D$ printing rapid prototyping technology for operation planning in revision lumbar discectomy. J Orthop Sci. 2015;20(3):475-80.

28. Kaya I, Cingöz ID, Șahin MC, Atar M, Ozyoruk S, Sayin M, Yuceer N. Are 3D Printing Templates an Advantage in Upper Thoracic Pedicle Screw Fixation? Cureus. 2021;13(3):e13989.

29. Sheth U, Theodoropoulos J, Abouali J. Use of 3-Dimensional Printing for Preoperative Planning in the Treatment of Recurrent Anterior Shoulder Instability. Arthrosc Tech. 2015;4(4):e311-6.

30. Liang H, Ji T, Zhang Y, Wang Y, Guo W. Reconstruction with 3D-printed pelvic endoprostheses after resection of a pelvic tumour. Bone Joint J. 2017;99-B(2):267-275.

31. Hunn SAM, Koefman AJ, Hunn AWM. 3D-printed Titanium Prosthetic Reconstruction of the C2 Vertebra: Techniques and Outcomes of Three Consecutive Cases. Spine (Phila Pa 1976). 2020;45(10):667-672.

32. Kadakia RJ, Wixted CM, Allen NB, Hanselman AE, Adams SB. Clinical applications of custom 3D printed implants in complex lower extremity reconstruction. 3D Print Med. 2020;6(1):29.

33. Zhang $\mathrm{C}$, Cao J, Zhu H, Fan H, Yang L, Duan X. Endoscopic Treatment of Symptomatic Foot and Ankle Bone Cyst with 3D Printing Application. Biomed Res Int. 2020;2020:8323658.

34. Starosolski ZA, Kan JH, Rosenfeld SD, Krishnamurthy R, Annapragada A. Application of 3-D printing (rapid prototyping) for creating physical models of pediatric orthopedic disorders. Pediatr Radiol. 2014;44(2):216-21.

35. Coote JD, Nguyen T, Tholen K, Stewart C, Verter E, McGee J, Celestre 
P, Sarkar K. Three-Dimensional Printed Patient Models for Complex Pediatric Spinal Surgery. Ochsner J. 2019;19(1):49-53.

36. Ren X, Yang L, Duan XJ. Three-dimensional printing in the surgical treatment of osteoid osteoma of the calcaneus: A case report. J Int Med Res. 2017;45(1):372-380.

37. Park JW, Kang HG, Kim JH, Kim HS. The application of 3D-printing technology in pelvic bone tumor surgery. J Orthop Sci. 2021;26(2):276283.

38. Gong S, Xu W, Wang R, Wang Z, Wang B, Han L, Chen G. Patientspecific instrumentation improved axial alignment of the femoral component, operative time and perioperative blood loss after total knee arthroplasty. Knee Surg Sports Traumatol Arthrosc. 2019;27(4):10831095.

39. Bonicoli E, Andreani L, Parchi P, Piolanti N, Lisanti M. Custom-fit total knee arthroplasty: our initial experience with 30 knees. Eur J Orthop Surg Traumatol. 2014;24(7):1249-54.

40. Nam D, Park A, Stambough JB, Johnson SR, Nunley RM, Barrack RL. The Mark Coventry Award: Custom Cutting Guides Do Not Improve Total Knee Arthroplasty Clinical Outcomes at 2 Years Followup. Clin Orthop Relat Res. 2016;474(1):40-6.

41. Hirao M, Ikemoto S, Tsuboi H, Akita S, Ohshima S, Saeki Y, Yoshikawa $\mathrm{H}$, Sugamoto K, Murase T, Hashimoto J. Computer assisted planning and custom-made surgical guide for malunited pronation deformity after first metatarsophalangeal joint arthrodesis in rheumatoid arthritis: a case report. Comput Aided Surg. 2014;19(1-3):13-9.

42. Bauer AS, Storelli DAR, Sibbel SE, McCarroll HR, Lattanza LL. Preoperative Computer Simulation and Patient-specific Guides are Safe and Effective to Correct Forearm Deformity in Children. J Pediatr Orthop. 2017;37(7):504-510.

43. Arnal-Burró J, Pérez-Mañanes R, Gallo-Del-Valle E, Igualada-Blazquez C, Cuervas-Mons M, Vaquero-Martín J. Three dimensional-printed patient-specific cutting guides for femoral varization osteotomy: Do it yourself. Knee. 2017;24(6):1359-1368.

44. IJpma FFA, Meesters AML, Merema BBJ, Ten Duis K, de Vries JPM, Banierink H, Wendt KW, Kraeima J, Witjes MJH. Feasibility of ImagingBased 3-Dimensional Models to Design Patient-Specific Osteosynthesis Plates and Drilling Guides. JAMA Netw Open. 2021;4(2):e2037519.

45. Lin CL, Fang JJ, Lin RM. Resection of giant invasive sacral schwannoma using image-based customized osteotomy tools. Eur Spine J. 2016;25(12):4103-4107.

46. Ma L, Zhou Y, Zhu Y, Lin Z, Wang Y, Zhang Y, Xia H, Mao C. 3D-printed guiding templates for improved osteosarcoma resection. Sci Rep. 2016;6:23335.

47. Li H, Qu X, Mao Y, Dai K, Zhu Z. Custom Acetabular Cages Offer Stable Fixation and Improved Hip Scores for Revision THA With Severe Bone Defects. Clin Orthop Relat Res. 2016;474(3):731-40.

48. Stoffelen DV, Eraly K, Debeer P. The use of 3D printing technology in reconstruction of a severe glenoid defect: a case report with 2.5 years of follow-up. J Shoulder Elbow Surg. 2015;24(8):e218-22.

49. Imanishi J, Choong PF. Three-dimensional printed calcaneal prosthesis following total calcanectomy. Int J Surg Case Rep. 2015;10:83-7.

50. Chandhanayingyong C, Srikong K, Puncreobutr C, Lohwongwatana B, Phimolsarnti R, Chuckpaiwong B. Three-dimensional printed, proximal phalangeal prosthesis with metatarsophalangeal joint arthroplasty for the treatment of a giant cell tumor of the fifth toe: The first case report. Int J Surg Case Rep. 2020;73:84-89.

51. Qiao F, Li D, Jin Z, Gao Y, Zhou T, He J, Cheng L. Application of $3 \mathrm{D}$ printed customized external fixator in fracture reduction. Injury. 2015;46(6):1150-5.

52. Raux S, Kohler R, Garin C, Cunin V, Abelin-Genevois K. Tridimensional trunk surface acquisition for brace manufacturing in idiopathic scoliosis. Eur Spine J. 2014;23 Suppl 4:S419-23.

53. Cobetto N, Aubin CE, Parent S, Clin J, Barchi S, Turgeon I, Labelle $\mathrm{H}$. Effectiveness of braces designed using computer-aided design and manufacturing (CAD/CAM) and finite element simulation compared to $\mathrm{CAD} / \mathrm{CAM}$ only for the conservative treatment of adolescent idiopathic scoliosis: a prospective randomized controlled trial. Eur Spine J. 2016;25(10):3056-3064.

54. Dombroski CE, Balsdon ME, Froats $A$. The use of a low cost 3D scanning and printing tool in the manufacture of custom-made foot orthoses: a preliminary study. BMC Res Notes. 2014;7:443.

55. Tanaka KS, Lightdale-Miric N. Advances in 3D-Printed Pediatric Prostheses for Upper Extremity Differences. J Bone Joint Surg Am. 2016;98(15):1320-6.

56. Swartz AQ, Turner K, Miller L, Kuiken T. Custom, rapid prototype thumb prosthesis for partial-hand amputation: A case report. Prosthet Orthot Int. 2018;42(2):187-190.

57. Gálvez JA, Gralewski K, McAndrew C, Rehman MA, Chang B, Levin LS. Assessment and Planning for a Pediatric Bilateral Hand Transplant Using 3-Dimensional Modeling: Case Report. J Hand Surg Am. 2016;41(3):341-3. 